\title{
Stromuling when stressed!
}

\author{
Björn Krenz ${ }^{1 *}$, Tai Wei Guo², Tatjana Kleinow ${ }^{3}$ \\ ${ }^{1}$ Lehrstuhl für Biochemie, Department Biologie, Staudtstr. 5, 91058 Erlangen, Germany \\ ${ }^{2}$ Department of Plant Pathology and Plant-Microbe Biology, Cornell University, Ithaca NY, USA \\ ${ }^{3}$ Institut für Biomaterialien und biomolekulare Systeme, Abteilung für Molekularbiologie und Virologie der Pflanzen, Universität Stuttgart, Pfaffenwaldring 57, 70550 Stuttgart, Germany
}

\begin{abstract}
Stromules are stroma-filled tubules, extruding from the plastid and surrounded by both envelope membranes, but so far, stromules remain enigmatic structures and their function unknown. Stromules can interconnect plastids and have been found to associate with the nucleus, endoplasmic reticulum, Golgi complex, plasma membrane, mitochondria and peroxisomes. This minireview briefly summarizes markers to visualize stromules, inducers of stromules and provides new data about plant virus induced stromules.
\end{abstract}

Keywords: stromule; geminivirus; plant virus; chloroplast

\section{Introduction}

Plastids are major and important organelles of the plant cell, as they perform essential biosynthetic and metabolic functions. These include photosynthetic carbon fixation, synthesis of amino acids, fatty acids, starch and secondary metabolites such as pigments [1]. There are several forms of differentiated plastids, i.e. chromoplast, leucoplast or chloroplast; and they are classified on the basis of their structure, pigment composition, metabolism and function.

Chloroplasts are characterized by their high concentration of chlorophyll and carry out a number of other functions besides photosynthesis, including fatty acid and amino acid synthesis [1], as well as immune response [2]. These organelles are not static, as they can circulate and move in plant cells when influenced by environmental factors, especially light. Cytoskeletal elements, such as actin, are involved in moving and positioning of chloroplasts [3].

Chloroplasts have two envelope membranes, the outer and inner chloroplast membranes, and are generally lens-shaped [4]. Protrusions emanating from chloroplasts are sometimes visible by light, fluorescence and electron microscopy. These thin, stroma-filled tubules, extruding from the plastid, and surrounded by both envelope membranes, are called stromules and have been observed for all plastid types examined so far [5-9]. Other organelles can have protrusions too, and corresponding terms were given to describe those extensions, according to the stromules for plastids convention:

\footnotetext{
* Corresponding author. Email: bjoern.krenz@fau.de
}

Handling Editor: Andrzej Bodył namely matrixules for mitochondria [10] and peroxules for peroxisomes [11]. Stromules are highly dynamic, branching and elongating across the plant cell, usually less than $1 \mu \mathrm{m}$ in diameter and of variable length. They can appear as short beak-like projections, to linear or branched structures up to several hundred $\mu \mathrm{m}$ long.

Stromules occur in all cell types, but stromule morphology and the proportion of plastids with stromules, vary from tissue to tissue and depend on plant developmental stages as well as biotic and abiotic stress conditions [12,13]. Although a lot of work has been done to understand stromules (reviewed in $[8,14]$ ), there are still a lot of unanswered questions. Here, we briefly summarize the current status on markers and inducers of stromules, with emphasis on plant pathogens as inducers.

\section{Stromule markers}

In addition to direct stromule observation by light and electron microscopy, or a fluorescence dye [15] in untransformed plants, a common method to visualize stromules in vivo is by the use of a marker protein fused with a fluorescent tag like the green fluorescent protein (GFP). One of the first markers applied was the CT-GFP construct $[16,17]$. This marker protein localizes to the chloroplast stroma and thereby also visualizes stromules.

Meanwhile, various constructs for stroma-targeted fluorescent marker proteins and also transgenic plant lines expressing these markers were established for plastid and stromule observation [18]. A transgenic Nicotiana benthamiana line, which is available to highlight the plastid 
stroma and stromules, expresses a GFP fusion protein with ferredoxin NADP $(\mathrm{H})$ oxidoreductase (FNR), FNR::eGFP [19-21]. Other constructs have also been reported to visualize stromules, e.g. chloroplast-localized heat shock cognate 70-kDa protein (cpHSC70-1) [22,23], outer envelope protein $16-2.1,-2.2$ and -1.3 [24] and mutants of the Arabidopsis resistance protein RPW8.2 [25].

Markers based on chloroplast outer envelope proteins have to be considered carefully. Breuers et al. [26] showed that stromule formation can simply be induced by overexpression of an outer envelope protein, e.g. outer envelope proteins AtLACS9 and AtTOC64-III. They showed that the formation is independent of the function or structure of the protein and posited that the proliferation of the membrane was a direct effect of the protein content in the membrane. So it must be taken into consideration that visualizing stromules usually requires protein overexpression, which might lead to artifacts. Therefore controls are necessary and essential. In general, expression of a stroma-targeted protein or a fluorescence dye in fluorescence microscopy analysis of stromules is more advisable than expression of a chloroplast membrane-localized protein.

\section{Stromule formation upon plant virus infection}

Kwok and Hanson [27] suggested that stromules may serve as pathways between nuclei, the cell periphery and possibly even other cells. They observed a concentration of plastids around nuclei with stromules reaching to the plasma membrane and through nuclear grooves. This close contact between plastids and the nuclei and/or the plasma membrane implied a function for the exchange of molecules between plastids and other organelles or diverse regions of the plant cell. Furthermore, Kwok and Hanson [27] found that stromules from two neighboring cells appeared to meet at either side of an adjoining cell wall. The cpHSC701-containing stromules detected upon an Abutilon mosaic virus (AbMV, plant ssDNA virus, Geminiviridae)-infection that was also in close association with the nucleus, appeared to interconnect plastids and extended from plastids outward to the cell periphery. This led to speculations of intra- and intercellular geminiviral transport in association with stromules [22,23]. A geminiviral nucleoprotein complex moves out of the plant nucleus via plasmodesmata into the adjacent cell to systemically infect the plant. Therefore we wanted to know, if stromules form structures spanning the nucleus, chloroplasts and plasmodesmata and if we can visualize an association of stromules and plasmodesmata. This association then could serve as an intracellular virus transport highway to the neighboring cell.

In our hands, stromule formation was never visualized by the overexpression of cpHSC70-1 alone, but only together with a plant DNA virus infection. Similar results were obtained with inoculation and co-expression experiments with AbMV and the Arabidopsis thaliana outer envelope protein 7 (OEP7; Fig. 1a). However, it was shown elsewhere that only the overexpression of OEP7 can lead to stromulelike structures formation [28]. OEP7 was fused to GFP and transiently expressed in $N$. benthamiana epidermal leaves.
OEP7::GFP signals were exclusively found in association with chloroplasts, but no stromules or stromule-like structures could be detected, except induced upon AbMV infection. Leaf material was also co-infiltrated with agrobacteria harboring an expression construct for the AbMV nuclear shuttle protein (NSP) fused to the red fluorescent protein (RFP). NSP is located in the plant nucleus, but additionally mobilized by the AbMV movement protein (MP) to the plasma membrane ([23] and references therein). NSP and MP together mediate cell-to-cell transfer and long distance transport of viral DNA replicated within the nucleus throughout the whole plant body. Thus, an AbMV-infected plant cell displays NSP::RFP signals in the nucleus and at the cell periphery.

In agreement with previous data, the OEP7::GFP marker also highlighted the induction of what appeared to be a stromule network upon an AbMV infection. What remains to be demonstrated is whether, under the condition of viral infection, the membranes of different plastids fuse with each other or if they just closely associate to each other. Chloroplasts tended to surround the nucleus and one could observe stromule formation connecting chloroplasts, along the nucleus or reaching towards the plasma membrane. Interestingly, stromules associated with the plasma membrane seemed to follow the membrane (Fig. 1c,d). Occasionally bulges along the stromules were observed. The OEP7::GFP labeled stromules induced by AbMV-infection exhibited similar appearance to those visualized by cpHSC70-1 [22,23].

In an additional experiment we wanted to test stromule association with plasmodesmata - microscopic channels that traverse the cell walls of plant cells [29]. Plasmodesmata enable direct, regulated, symplastic intercellular transport of substances between cells. The 3 a movement protein (MP) of cucumber mosaic virus (CMV) targets plasmodesmata and accumulates in the central cavity of the pore [30] and was used in this assay as a marker protein for plasmodesmata. CMV MP was fused with RFP and co-expressed with OEP7::GFP and AbMV. CMV MP::RFP highlighted plasmodesmata, and again, AbMV infection was sufficient to induce stromules visualized by OEP7::GFP, but we could never observe an association of CMV MP::RFP and OEP7::GFP in this experimental set-up. Also the bulges or packet structures along the stromules were never associated with the plasmodesmata marker (data not shown).

\section{Inducers of stromules}

Schattat et al. [14,31,32] do not support a function of stromules in trafficking of macromolecules between plastids, despite the strong microscopic impression of interplastid connectivity via stromules. They could not observe an exchange of a stroma marker protein between independent plastids. Hanson and Sattarzadeh [33] then commented and showed evidence for the flow of proteins between interconnected plastids. They claimed that Schattat et al. [14] were unable to observe movement because of stromule breakage or disruption of the fluorescent protein by the high-intensity laser power they employed. Mathur and Barton [34] disagreed with this explanation, stating that their procedure was very mild in comparison to procedures using intense 
lasers. While the groups disagree about whether proteins can flow between plastids already connected with stromules, they both agree that the debate surrounding the formation of new connections between separate plastids will continue until a stromule in the act of attaching to another one is

Beside possible interplastid connectivity via stromules, it is striking that stromules or stromule-like structures also extend to the plasma membrane, as well as the nucleus. It may indicate a specific response to viral infection resulting in increased communication between chloroplasts and other cell compartments, or a more general stress response of the cell. Various abiotic and biotic stress conditions including lular sucrose or glucose [21], colonization by an arbuscular mycorrhizal fungus [36] and infiltration of agrobacteria [31] were described as inducers of stromules. Gray et al. [37] showed that various stress treatments, including drought and salt stress, are able to induce stromule formation in tobacco epidermal cells. Application of abscisic acid (ABA) to tobacco found during a photoconversion experiment. heat [9], subcellular redox stress [35], application of extracel-

and wheat seedlings also induced stromule formation very effectively. Stromules were more abundant in dark-grown seedlings than in light-grown seedlings, and stromule formation was sensitive to red and far-red light. Stromules were induced by treatment with the ethylene precursor ACC (1-aminocyclopropane-1-carboxylic acid) and by treatment with methyl jasmonate.

Undoubtedly, stromule formation can be induced by plant viruses [22,23,38-40] (and this study). RNA-virus infected sugar beets showed structures resembling stromules [40]. Shalla [38] described stromule occurrence in TMV-infected tomatoes. Caplan and colleagues described the same in TMV-infected tobacco plants [39], just as for N. benthamiana plants locally infected with the AbMV [22,23]. Several geminiviruses were found to interact with diverse plant hormone pathways, such as the salicylic acid, ethylene, jasmonic acid pathways and to the brassinosteroid pathway. They activate the salicylic acid and ethylene pathways, which both participate in the host defense response [41]. Transcriptomic analysis has recently shown that geminiviruses
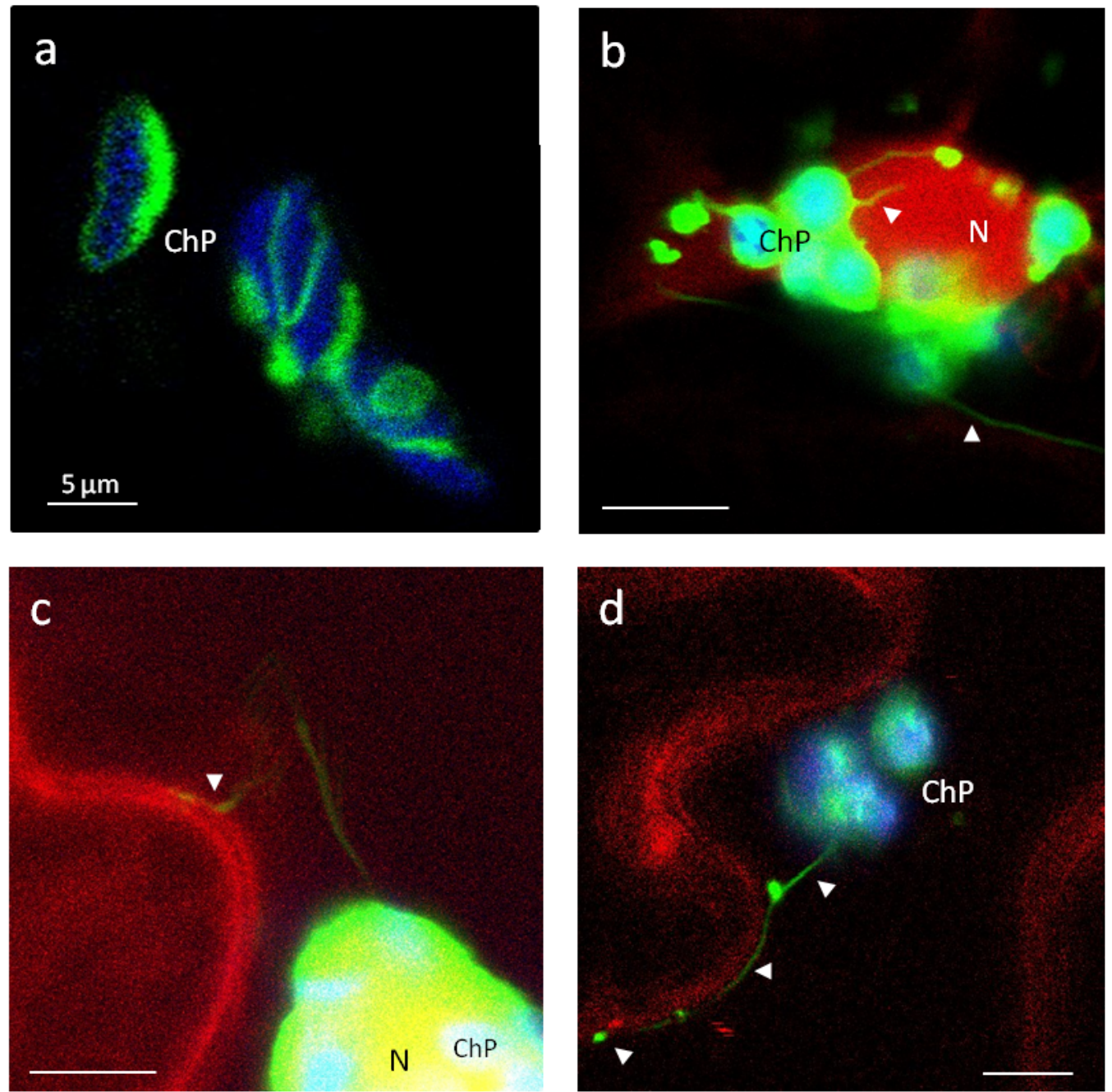

Fig. 1 Abutilon mosaic virus-induced OEP7-containing stromules extending from plastids to the nucleaus and cell periphery. Transient co-expression of test constructs in leaf tissues of non-infected and locally AbMV-infected N. benthamiana and confocal fluorescence microscopy. AbMV infection was established by simultaneous agro-infiltration of infectious DNA A and DNA B clones with the fluorescent protein expression constructs. a Merged image of chloroplast (ChP) with full length OEP7:green fluorescent protein (GFP) driven under the CaMV 35S promotor in non-infected plants, chloroplast autofluorescence is shown in blue, 2dpi. b-d Merged image of chloroplasts (ChP) and nucleus (N) with OEP7:green fluorescent protein (GFP) and NSP:red fluorescent protein (RFP) in AbMV-infected cells, NSP::RFP marks nucleus and plasma membrane, chloroplast autofluorescence is shown in blue, 2dpi. Arrowheads highlight stromules. Bar: $5 \mu \mathrm{m}$. 
also induce ABA- and ethylene responsive genes in tomato [42]. So there is the possibility that geminiviruses induce stromules by interfering with the plant hormone signaling. But if stromule formation is part of an antiviral immune response then it either comes too late or the virus has the capability to neutralize the stress response, irrespective of stromule formation.

\section{Signaling molecules and stromule function?}

Although the function of stromules is unknown, a putative correlation between plastid metabolic activity and stromule biogenesis has been proposed, enabling increased molecular exchange between plastids and other organelles or adjacent cells to process environmental stimuli [17,18,27,33]. The identity of proteins, metabolites and other signaling components, which may be transported are unknown, but there are data indicating that some molecules are excluded. Thylakoid membranes, plastid DNA, and ribosomes have not been detected within stromules [43], while Rubisco and aspartate aminotransferase have been observed [44].

Newell et al. [43] tried to determine if plastid DNA or plastid ribosomes are able to enter stromules, potentially permitting the transfer of genetic information between plastids. Plastid DNA and ribosomes were marked with green fluorescent protein (GFP) fusions to LacI, the lac repressor, and their observations indicated that plastid DNA and plastid ribosomes do not routinely move into stromules in tobacco and Arabidopsis. Nevertheless, the possibility of a

\section{Acknowledgments}

We would like to acknowledge the Optical Imaging Centre Erlangen (OICE). We are also grateful to Dr. Christopher E. Lane for critically reading of the manuscript. This work was partially financed by the CRC796, Erlangen.

\section{Authors' contributions}

The following declarations about authors' contributions to the research have been made: performed the experiments: TWG; designed the experiments and wrote the manuscript: BK, TK.

\section{Competing interests}

No competing interests have been declared.

\section{References}

1. Rolland N, Curien G, Finazzi G, Kuntz M, Maréchal E, Matringe $\mathrm{M}$, et al. The biosynthetic capacities of the plastids and integration between cytoplasmic and chloroplast processes. Annu Rev Genet. 2012;46(1):233-264. http://dx.doi.org/10.1146/ annurev-genet-110410-132544

2. Stael S, Kmiecik P, Willems P, van der Kelen K, Coll NS, Teige M, et al. Plant innate immunity - sunny side up? Trends Plant Sci. 2014 (in press). http://dx.doi.org/10.1016/j.tplants.2014.10.002

3. Oikawa K, Kasahara M, Kiyosue T, Kagawa T, Suetsugu N, Takahashi F, et al. CHLOROPLAST UNUSUAL POSITIONING1 is essential for proper chloroplast positioning. Plant Cell. 2003;15(12):2805-2815. http://dx.doi.org/10.1105/tpc.016428

4. Cavalier-Smith T. Membrane heredity and early chloroplast evolution. Trends Plant Sci. 2000;5(4):174-182. http://dx.doi.org/10.1016/ S1360-1385(00)01598-3

5. Köhler RH, Hanson MR. Plastid tubules of higher plants are tissue-specific and developmentally regulated. J Cell Sci. 2000;113 (pt 1):81-89. rare movement of plastid DNA and ribosomes or the transfer of much smaller DNA molecules, e.g., plastid transformation vectors, via stromules cannot be completely excluded. We observed stromules associated along a stretch of plasma membrane (Fig. 1c,d) and a possible explanation for this phenomenon is that the intimate association of plasma membrane and stromule provides a connection for rapid exchange of membrane lipids or other molecules. But this is rather speculative, so far.

Stromules are also discussed as structures supporting the autophagy of plastid material [45]. Reactive oxygen species lead to the fragmentation and oxidation of stroma proteins, which then bud to form Rubisco-containing bodies (RCB). Autophagosomes drive RCBs then to the vacuole for degradation [46].

\section{Future directions}

The biggest challenge in the future will be to decipher the function of stromules and the molecular mechanisms in stromule formation. Although numerous stromule markers and inducers are known, there is no stromule mutant available, which would be a huge step in understanding stromules. It is still unclear if stromules act as interaction hubs between subcellular compartments or cells and if molecules are trafficking. There is reasonable doubt that these are stroma-localized proteins, but maybe outer membrane envelope proteins, $\mathrm{Ca}^{2+}$ or lipids? We are curious about the first answers in the stromule riddle.

6. Hanson MR, Köhler RH. GFP imaging: methodology and application to investigate cellular compartmentation in plants. J Exp Bot. 2001;52(356):529-539. http://dx.doi.org/10.1093/jexbot/52.356.529

7. Sage TL, Sage RF. The functional anatomy of rice leaves: implications for refixation of photorespiratory $\mathrm{CO}_{2}$ and efforts to engineer $\mathrm{C}_{4}$ photosynthesis into rice. Plant Cell Physiol. 2009;50(4):756-772. http://dx.doi.org/10.1093/pcp/pcp033

8. Mathur J, Mammone A, Barton KA. Organelle extensions in plant cells. J Integr Plant Biol. 2012;54:851-867. http://dx.doi. org/10.1111/j.1744-7909.2012.01175.x

9. Holzinger A, Buchner O, Lütz C, Hanson MR. Temperature-sensitive formation of chloroplast protrusions and stromules in mesophyll cells of Arabidopsis thaliana. Protoplasma. 2007;230(1-2):23-30. http:// dx.doi.org/10.1007/s00709-006-0222-y

10. Logan DC. The mitochondrial compartment. J Exp Bot. 2006;57(6):1225-1243. http://dx.doi.org/10.1093/jxb/erj151

11. Bishop GJ. Refining the plant steroid hormone biosynthesis pathway. Trends Plant Sci. 2007;12(9):377-380. http://dx.doi.org/10.1016/j. tplants.2007.07.001

12. Gunning BES. Plastid stromules: video microscopy of their outgrowth, retraction, tensioning, anchoring, branching, bridging, and tip-shedding. Protoplasma. 2005;225(1-2):33-42. http://dx.doi. org/10.1007/s00709-004-0073-3

13. Waters MT, Fray RG, Pyke KA. Stromule formation is dependent upon plastid size, plastid differentiation status and the density of plastids within the cell. Plant J. 2004;39(4):655-667. http://dx.doi. org/10.1111/j.1365-313X.2004.02164.x

14. Schattat MH, Barton KA, Mathur J. The myth of interconnected plastids and related phenomena. Protoplasma. 2014 (in press). http:// dx.doi.org/10.1007/s00709-014-0666-4

15. Menzel D. An interconnected plastidom in Acetabularia: implications 
for the mechanism of chloroplast motility. Protoplasma. 1994;179(34):166-171. http://dx.doi.org/10.1007/BF01403955

16. Köhler RH, Zipfel WR, Webb WW, Hanson MR. The green fluorescent protein as a marker to visualize plant mitochondria in vivo. Plant J. 1997;11(3):613-621. http://dx.doi. org/10.1046/j.1365-313X.1997.11030613.x

17. Köhler RH, Cao J, Zipfel WR, Webb WW, Hanson MR. Exchange of protein molecules through connections between higher plant plastids. Science. 1997;276(5321):2039-2042. http://dx.doi.org/10.1126/ science. 276.5321 .2039

18. Natesan SKA, Sullivan JA, Gray JC. Stromules: a characteristic cell-specific feature of plastid morphology. J Exp Bot. 2005;56(413):787-797. http://dx.doi.org/10.1093/jxb/eri088

19. Erickson JL, Ziegler J, Guevara D, Abel S, Klösgen RB, Mathur J, et al. Agrobacterium-derived cytokinin influences plastid morphology and starch accumulation in Nicotiana benthamiana during transient assays. BMC Plant Biol. 2014;14(1):127. http://dx.doi. org/10.1186/1471-2229-14-127

20. Marques JP. In vivo transport of folded EGFP by the pH/TATdependent pathway in chloroplasts of Arabidopsis thaliana. J Exp Bot. 2004;55(403):1697-1706. http://dx.doi.org/10.1093/jxb/erh191

21. Schattat M, Klösgen R. Induction of stromule formation by extracellular sucrose and glucose in epidermal leaf tissue of Arabidopsis thaliana. BMC Plant Biol. 2011;11(1):115. http://dx.doi. org/10.1186/1471-2229-11-115

22. Krenz B, Windeisen V, Wege C, Jeske H, Kleinow T. A plastid-targeted heat shock cognate $70 \mathrm{kDa}$ protein interacts with the Abutilon mosaic virus movement protein. Virology. 2010;401(1):6-17. http://dx.doi. org/10.1016/j.virol.2010.02.011

23. Krenz B, Jeske H, Kleinow T. The induction of stromule formation by a plant DNA-virus in epidermal leaf tissues suggests a novel intraand intercellular macromolecular trafficking route. Front Plant Sci. 2012;3:291. http://dx.doi.org/10.3389/fpls.2012.00291

24. Mueller SJ, Lang D, Hoernstein SNW, Lang EGE, Schuessele C, Schmidt A, et al. Quantitative analysis of the mitochondrial and plastid proteomes of the moss Physcomitrella patens reveals protein macrocompartmentation and microcompartmentation. Plant Physiol. 2014;164(4):2081-2095. http://dx.doi.org/10.1104/pp.114.235754

25. Wang W, Zhang Y, Wen Y, Berkey R, Ma X, Pan Z, et al. A comprehensive mutational analysis of the Arabidopsis resistance protein RPW8.2 reveals key amino acids for defense activation and protein targeting. Plant Cell. 2013;25(10):4242-4261. http://dx.doi.org/10.1105/ tpc.113.117226

26. Breuers FKH, Bräutigam A, Geimer S, Welzel UY, Stefano G, Renna $\mathrm{L}$, et al. Dynamic remodeling of the plastid envelope membranes - a tool for chloroplast envelope in vivo localizations. Front Plant Sci. 2012;3:7. http://dx.doi.org/10.3389/fpls.2012.00007

27. Kwok EY, Hanson MR. Plastids and stromules interact with the nucleus and cell membrane in vascular plants. Plant Cell Rep. 2004;23(4):188195. http://dx.doi.org/10.1007/s00299-004-0824-9

28. Machettira AB, Groß LE, Tillmann B, Weis BL, Englich G, Sommer MS, et al. Protein-induced modulation of chloroplast membrane morphology. Front Plant Sci. 2012;2:118. http://dx.doi.org/10.3389/ fpls.2011.00118

29. Oparka KJ. Getting the message across: how do plant cells exchange macromolecular complexes? Trends Plant Sci. 2004;9(1):33-41. http:// dx.doi.org/10.1016/j.tplants.2003.11.001

30. Blackman LM, Boevink P, Cruz SS, Palukaitis P, Oparka KJ. The movement protein of cucumber mosaic virus traffics into sieve elements in minor veins of Nicotiana clevelandii. Plant Cell. 1998;10(4):525-538.
31. Schattat MH, Griffiths S, Mathur N, Barton K, Wozny MR, Dunn N, et al. Differential coloring reveals that plastids do not form networks for exchanging macromolecules. Plant Cell. 2012;24(4):1465-1477. http://dx.doi.org/10.1105/tpc.111.095398

32. Schattat MH, Klösgen RB, Mathur J. New insights on stromules: stroma filled tubules extended by independent plastids. Plant Signal Behav. 2012;7(9):1132-1137. http://dx.doi.org/10.4161/psb.21342

33. Hanson MR, Sattarzadeh A. Trafficking of proteins through plastid stromules. Plant Cell. 2013;25(8):2774-2782. http://dx.doi.org/10.1105/ tpc. 113.112870

34. Mathur J, Barton KA, Schattat MH. Fluorescent protein flow within stromules. Plant Cell. 2013;25(8):2771-2772. http://dx.doi.org/10.1105/ tpc.113.117416

35. Itoh RD, Yamasaki H, Septiana A, Yoshida S, Fujiwara MT. Chemical induction of rapid and reversible plastid filamentation in Arabidopsis thaliana roots. Physiol Plant. 2010;139(2):144-158. http://dx.doi. org/10.1111/j.1399-3054.2010.01352.x

36. Lohse S. Organization and metabolism of plastids and mitochondria in arbuscular mycorrhizal roots of Medicago truncatula. Plant Physiol. 2005;139(1):329-340. http://dx.doi.org/10.1104/pp.105.061457

37. Gray JC, Hansen MR, Shaw DJ, Graham K, Dale R, Smallman P, et al. Plastid stromules are induced by stress treatments acting through abscisic acid: stress induction of plastid stromules. Plant J. 2012;69(3):387398. http://dx.doi.org/10.1111/j.1365-313X.2011.04800.x

38. Shalla TA. Assembly and aggregation of tobacco mosaic virus in tomato leaflets. J Cell Biol. 1964;21:253-264.

39. Caplan JL, Mamillapalli P, Burch-Smith TM, Czymmek K, DineshKumar SP. Chloroplastic protein NRIP1 mediates innate immune receptor recognition of a viral effector. Cell. 2008;132(3):449-462. http://dx.doi.org/10.1016/j.cell.2007.12.031

40. Esau K. Anatomical and cytological studies on beet mosaic. J Agric Res. 1944;69:95-117.

41. Ascencio-Ibanez JT, Sozzani R, Lee TJ, Chu TM, Wolfinger RD, Cella $\mathrm{R}$, et al. Global analysis of Arabidopsis gene expression uncovers a complex array of changes impacting pathogen response and cell cycle during geminivirus infection. Plant Physiol. 2008;148(1):436-454. http://dx.doi.org/10.1104/pp.108.121038

42. Miozzi L, Napoli C, Sardo L, Accotto GP. Transcriptomics of the interaction between the monopartite phloem-limited geminivirus tomato yellow leaf curl Sardinia virus and Solanum lycopersicum highlights a role for plant hormones, autophagy and plant immune system fine tuning during infection. PLoS ONE. 2014;9(2):e89951. http://dx.doi.org/10.1371/journal.pone.0089951

43. Newell CA, Natesan SKA, Sullivan JA, Jouhet J, Kavanagh TA, Gray JC. Exclusion of plastid nucleoids and ribosomes from stromules in tobacco and Arabidopsis: absence of nucleoids and ribosomes from stromules. Plant J. 2012;69(3):399-410. http://dx.doi. org/10.1111/j.1365-313X.2011.04798.x

44. Kwok EY. GFP-labelled Rubisco and aspartate aminotransferase are present in plastid stromules and traffic between plastids. J Exp Bot. 2004;55(397):595-604. http://dx.doi.org/10.1093/jxb/erh062

45. Ishida H, Yoshimoto K, Izumi M, Reisen D, Yano Y, Makino A, et al. Mobilization of rubisco and stroma-localized fluorescent proteins of chloroplasts to the vacuole by an ATG gene-dependent autophagic process. Plant Physiol. 2008;148(1):142-155. http://dx.doi.org/10.1104/ pp.108.122770

46. Avila-Ospina L, Moison M, Yoshimoto K, Masclaux-Daubresse C. Autophagy, plant senescence, and nutrient recycling. J Exp Bot. 2014;65(14):3799-3811. http://dx.doi.org/10.1093/jxb/eru039 